\title{
Current-induced spin polarization in spin-orbit-coupled electron systems
}

\author{
Ming-Hao Liu (劉明豪), * Son-Hsien Chen (陳松賢), and Ching-Ray Chang (張慶瑞) \\ Department of Physics, National Taiwan University, Taipei 10617, Taiwan \\ (Received 25 February 2008; revised manuscript received 29 July 2008; published 20 October 2008)
}

\begin{abstract}
Current-induced spin polarization (CISP) is rederived in ballistic spin-orbit-coupled electron systems, based on equilibrium statistical mechanics. A simple and useful picture is correspondingly proposed to help understand the CISP and predict the polarization direction. Nonequilibrium Landauer-Keldysh formalism is applied to demonstrate the validity of the statistical picture, taking the linear Rashba-Dresselhaus [001] twodimensional system as a specific example. Spin densities induced by the CISP in semiconductor heterostructures and in metallic surface states are compared, showing that the CISP increases with the spin-splitting strength, and hence suggesting that the CISP should be more observable on metal and semimetal surfaces due to the discovered strong Rashba splitting. An application of the CISP designed to generate a spin-Hall pattern in the in-plane, instead of the out-of-plane, component is also proposed.
\end{abstract}

DOI: 10.1103/PhysRevB.78.165316

PACS number(s): 72.25.Pn, 71.70.Ej, 85.75.-d

\section{INTRODUCTION}

The aim of preparing and controlling spins in all-electrical nonmagnetic devices has been shown to be possible in semiconducting bulk and two-dimensional electron systems (2DESs). ${ }^{1,2}$ Besides the optical spin injection, a much more natural way of spin orientation is to make use of the spinorbit (SO) coupling due to the lack of inversion symmetry of the underlying material. ${ }^{3}$ When passing an unpolarized electric current (electrons carrying random spins) through an SOcoupled material, spin-dependent consequences arise, among which two famous phenomena are the spin-Hall effect (SHE) (Refs. 4-9) and the current-induced spin polarization (CISP).

In the CISP phenomenon, unpolarized electric current is expected to be spin polarized when flowing in a SO-coupled sample. This effect was first theoretically proposed in the early 90 s. Edelstein ${ }^{10}$ employed linear-response theory to calculate the spin polarization due to an electric current in the presence of SO coupling linear in momentum, taking into account low-concentration impurities. Aronov and Lyanda-Geller ${ }^{11}$ solved the quantum Liouville's theorem for the spin-density matrix to show the CISP, taking into account scattering as well. Recently, the CISP phenomenon has been experimentally proven. ${ }^{12-14}$ Moreover, both the SHE and CISP have been observed at room temperature..$^{15}$

In this paper we propose another viewpoint based on equilibrium statistical mechanics to explain the CISP in the absence of impurity scattering for both bulk and twodimensional systems. We show that the canonical ensemble average (CEA) of electrons moving with a wave vector $\mathbf{k}$ immediately prescribes a spin polarization antiparallel to the effective magnetic field $\mathbf{B}_{\text {eff }}(\mathbf{k})$ stemming from the underlying SO coupling not necessarily linear in $k$, and hence explains the CISP. Correspondingly, a much simpler picture, compared to the early theoretical works of Refs. 10 and 11, helps provide a qualitative and straightforward explanation for the CISP: In an SO-coupled 2DES without external magnetic field, an ensemble of rest electrons is unpolarized while it becomes spin-polarized antiparallel to $\mathbf{B}_{\text {eff }}(\mathbf{k})$ when moving along $\mathbf{k}$ (see Fig. 1).

To demonstrate the validness of this elementary statistical argument, spin and charge transports in finite-size four- terminal conducting 2DESs with Rashba and linear Dresselhaus [001] SO couplings are numerically analyzed using the more sophisticated Landauer-Keldysh formalism (LKF), ${ }^{16-18}$ allowing for nonequilibrium statistics. Good agreement between the analytical CEA and the numerical LKF will be seen, consolidating our statistical picture. In addition to the semiconducting heterostructures, we also extend the analysis of the CISP to metal and semimetal surfaces, and compare the polarization strengths. Finally, an application of the CISP, resembling an in-plane SHE, will be subsequently proposed. Throughout this paper, all the band parameters used in the LKF are extracted from experiments by matching the band structures calculated by the tight-binding model (and hence the density of states calculated by the LKF) with the experimentally measured ones. ${ }^{19}$

This paper is organized as follows. In Sec. II, we discuss the general properties of the system with SO coupling and derive the CISP in the ballistic limit using statistical mechanics. In Sec. III the LKF is applied partly to examine the validity of the statistical picture of the CISP introduced in Sec. II, and partly for further investigation. Summary of the present work will be given in Sec. IV.

\section{ANALYTICAL DERIVATIONS}

Consider a SO-coupled system, subject to the singleparticle Hamiltonian

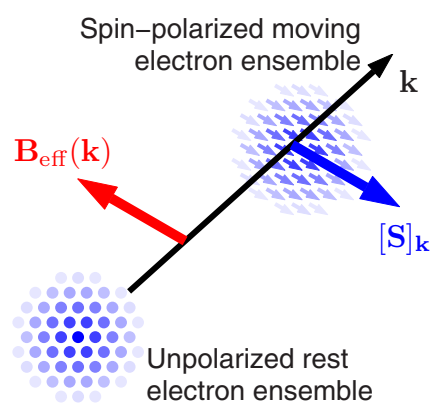

FIG. 1. (Color online) Statistical picture of the current-induced spin polarization phenomenon. 


$$
\mathcal{H}=\frac{\hbar^{2} \mathbf{k}^{2}}{2 m} \mathbb{1}+\mathbf{S} \cdot \vec{\Omega}(\mathbf{k}),
$$

where $m$ is the effective mass, 1 is the $2 \times 2$ identity matrix, $\mathbf{S}=(\hbar / 2) \vec{\sigma}$ is the spin operator, $\vec{\sigma} \equiv\left(\sigma^{x}, \sigma^{y}, \sigma^{z}\right)$ being the Pauli-matrix vector, and $\vec{\Omega}(\mathbf{k})=(e / m c) \mathbf{B}_{\text {eff }}(\mathbf{k})$ is the momentum-dependent Larmor frequency vector, with $\mathbf{B}_{\text {eff }}(\mathbf{k})$ being the effective magnetic field stemming from the SO coupling. ${ }^{20}$

\section{A. Larmor frequency vectors}

For III-V (zinc blende) bulk semiconductors, ${ }^{21}$ the Larmor frequency in Eq. (1) is written as ${ }^{22}$

$$
\vec{\Omega}(\mathbf{k})=\frac{\eta \hbar^{2}}{\left(2 m^{3} E_{g}\right)^{1 / 2}} \vec{\kappa},
$$

where $\eta$ is a dimensionless parameter specifying the spinorbit coupling strength, $E_{g}$ is the band gap, and $\vec{\kappa}$ is given by

$$
\vec{\kappa}=\left(\begin{array}{l}
k_{x}\left(k_{y}^{2}-k_{z}^{2}\right) \\
k_{y}\left(k_{z}^{2}-k_{x}^{2}\right) \\
k_{z}\left(k_{x}^{2}-k_{y}^{2}\right)
\end{array}\right) .
$$

Here $k_{i}$ 's are the wave vector components along the crystal principle axes.

When restricted to two-dimension, the component of the wave vector normal to the 2DES is averaged. For [001] quantum wells, one has $k_{z}^{2} \rightarrow\left\langle k_{z}^{2}\right\rangle$ and $k_{z} \rightarrow\left\langle k_{z}\right\rangle=\left\langle i \partial_{z}\right\rangle=0$ to rewrite Eq. (3) as $\vec{k}^{[001]}=\left[k_{x}\left(k_{y}^{2}-\left\langle k_{z}^{2}\right\rangle\right), k_{y}\left(\left\langle k_{z}^{2}\right\rangle-k_{x}^{2}\right), 0\right]$, so that the Larmor frequency (2) takes the form

$$
\vec{\Omega}^{[001]}=\frac{2 \beta}{\hbar}\left(-k_{x}, k_{y}, 0\right)+\frac{2 \beta}{\hbar\left\langle k_{z}^{2}\right\rangle}\left(k_{x} k_{y}^{2},-k_{y} k_{x}^{2}, 0\right),
$$

where $\beta$ is defined by

$$
\beta=\frac{\hbar}{2} \frac{\eta \hbar^{2}}{\left(2 m^{3} E_{g}\right)^{1 / 2}}\left\langle k_{z}^{2}\right\rangle=\gamma\left\langle k_{z}^{2}\right\rangle,
$$

and is referred to as the Dresselhaus SO coupling constant. The $\gamma$ parameter (corresponding to $\mathfrak{b}_{41}^{6 c 6 c}$ of Ref. 3 ) is material dependent, and is roughly $27 \mathrm{eV} \AA^{3}$ for both GaAs and InAs. ${ }^{3,23}$

The first term in Eq. (4),

$$
\vec{\Omega}_{D}^{[001]}=\frac{2 \beta}{\hbar}\left(-k_{x}, k_{y}, 0\right),
$$

is the linear Dresselhaus [001] term, which will dominate for small $k$ region. The corresponding SO term $\mathcal{H}_{D}^{[001]}$ $=\mathbf{S} \cdot \Omega_{D}^{[001]}=\beta\left(-k_{x} \sigma^{x}+k_{y} \sigma^{y}\right)$ is known as the linear Dresselhaus [001] model Hamiltonian. ${ }^{3,20}$ With larger $k$ the second term in Eq. (4)-the $k^{3}$ term-becomes important. We will come back to this later. For other quantum wells such as [110] and [111], the $\vec{\kappa}$ vector given by Eq. (3) can be recast into a form that depends on the growth direction $\hat{n}$ of the 2DES. ${ }^{24}$ (See also Ref. 20.)

When writing the Larmor frequency vector as

$$
\vec{\Omega}_{R}=\frac{2 \alpha}{\hbar}(\mathbf{k} \times \hat{n}),
$$

the linear Rashba model Hamiltonian ${ }^{3,20,25} \mathcal{H}_{R}=\mathbf{S} \cdot \vec{\Omega}_{R}=\alpha(\mathbf{k}$ $\times \hat{n})$ is recovered. Here $\alpha$ is the Rashba SO coupling constant.

\section{B. Time-reversal symmetry}

Before deriving the CISP, we provide the following two intrinsic properties of the Hamiltonian (1). First, we show that the contribution to the SO terms in solid is odd in $k$ due to time-reversal symmetry, which is also remarked in Ref. 3. For spin-1/2 systems subject to Hamiltonian (1), the energy dispersion can be written as

$$
E_{\sigma}(\mathbf{k})=E_{0}+\sigma \Delta_{\mathbf{k}},
$$

where $E_{0}=\hbar^{2} k^{2} / 2 m$ is the kinetic energy, $\sigma= \pm 1$ is the spin state label, and $\Delta_{\mathbf{k}}$ is the spin splitting due to SO coupling. In the absence of external magnetic field, the time-reversal symmetry is preserved, resulting in $E_{+}(\mathbf{k})=E_{-}(-\mathbf{k})$ or

$$
+\Delta_{\mathrm{k}}=-\Delta_{-\mathbf{k}},
$$

which implies that nonvanishing spin splitting $\Delta_{\mathbf{k}}$ is odd in $k$. Note that Eq. (9) also implies

$$
\vec{\Omega}(-\mathbf{k})=-\vec{\Omega}(\mathbf{k}),
$$

which agrees with our intuition. Apparently, Eq. (10) is obeyed by all the previously reviewed Larmor frequency vectors.

Second, we show $\langle \pm, \mathbf{k}|\vec{\sigma}| \pm, \mathbf{k}\rangle=-\langle\mp, \mathbf{k}|\vec{\sigma}| \mp, \mathbf{k}\rangle$, where $|\sigma, \mathbf{k}\rangle$ is the eigenstate of Hamiltonian (1). We begin with the Schrödinger equation,

$$
\mathcal{H}|\sigma, \mathbf{k}\rangle=\left(\frac{\hbar^{2} \mathbf{k}^{2}}{2 m} 1+\mathbf{S} \cdot \vec{\Omega}(\mathbf{k})\right)|\sigma, \mathbf{k}\rangle=E_{\sigma}(\mathbf{k})|\sigma, \mathbf{k}\rangle
$$

Comparing Eq. (11) with Eq. (8), we deduce $\mathbf{S} \cdot \vec{\Omega}(\mathbf{k})|\sigma, \mathbf{k}\rangle$ $=\sigma \Delta_{\mathbf{k}}|\sigma, \mathbf{k}\rangle$ or

$$
\langle\sigma, \mathbf{k}|\mathbf{S} \cdot \vec{\Omega}(\mathbf{k})| \sigma, \mathbf{k}\rangle=\sigma \Delta_{\mathbf{k}},
$$

where $|\sigma, \mathbf{k}\rangle$ is assumed normalized. This implies

$$
\langle+, \mathbf{k}|\mathbf{S} \cdot \vec{\Omega}(\mathbf{k})|+, \mathbf{k}\rangle=-\langle-, \mathbf{k}|\mathbf{S} \cdot \vec{\Omega}(\mathbf{k})|-, \mathbf{k}\rangle .
$$

Factoring out and canceling $\vec{\Omega}(\mathbf{k})$ on both sides, we arrive at

$$
\langle+, \mathbf{k}|\vec{\sigma}|+, \mathbf{k}\rangle=-\langle-, \mathbf{k}|\vec{\sigma}|-, \mathbf{k}\rangle .
$$

Equation (14) is a general property of Eq. (1) and is valid for systems with dispersions $E_{\sigma}(\mathbf{k})=E_{0}+\sigma \Delta_{\mathbf{k}}$, where the spin splitting $\Delta_{\mathbf{k}}$ is not necessarily linear in $k$. This property (14) will play a tricky role in the coming derivation of the CISP based on statistical mechanics in Sec. II C.

Note that Eq. (14) is also a consequence of time-reversal symmetry (9), as one can easily prove as follows. Using Eq. (12) we rewrite Eq. (9) as

$$
\langle+, \mathbf{k}|\mathbf{S} \cdot \vec{\Omega}(\mathbf{k})|+, \mathbf{k}\rangle=\langle-,-\mathbf{k}|\mathbf{S} \cdot \vec{\Omega}(-\mathbf{k})|-,-\mathbf{k}\rangle .
$$

Equation (12) also implies 


$$
\langle\sigma, \mathbf{k}|\mathbf{S} \cdot \vec{\Omega}(\mathbf{k})| \sigma, \mathbf{k}\rangle=-\langle-\sigma, \mathbf{k}|\mathbf{S} \cdot \vec{\Omega}(\mathbf{k})|-\sigma, \mathbf{k}\rangle
$$

when one regards $\sigma \Delta_{\mathbf{k}}$ as $-(-\sigma) \Delta_{\mathbf{k}}$. In addition, Eq. (9) implies

$$
\langle\sigma, \mathbf{k}|\mathbf{S} \cdot \vec{\Omega}(\mathbf{k})| \sigma, \mathbf{k}\rangle=\langle\sigma,-\mathbf{k}|\mathbf{S} \cdot \vec{\Omega}(\mathbf{k})| \sigma,-\mathbf{k}\rangle
$$

because of

$$
\begin{aligned}
\langle\sigma, \mathbf{k}|\mathbf{S} \cdot \vec{\Omega}(\mathbf{k})| \sigma, \mathbf{k}\rangle \\
\quad=\sigma \Delta_{\mathbf{k}} \\
=-\sigma \Delta_{-\mathbf{k}} \\
=\langle-\sigma,-\mathbf{k}|\mathbf{S} \cdot \vec{\Omega}(-\mathbf{k})|-\sigma,-\mathbf{k}\rangle \\
=-\langle\sigma,-\mathbf{k}|\mathbf{S} \cdot \vec{\Omega}(-\mathbf{k})| \sigma,-\mathbf{k}\rangle \\
=\langle\sigma,-\mathbf{k}|\mathbf{S} \cdot \vec{\Omega}(\mathbf{k})| \sigma,-\mathbf{k}\rangle,
\end{aligned}
$$

where Eqs. (16) and (10) are used in (18a) and (18b), respectively. Substituting Eqs. (10) and (17) into Eq. (15), we obtain Eq. (13), and hence the property (14).

\section{Current-induced spin polarization by canonical ensemble average}

Having seen the general properties of the Hamiltonian (1) under the time-reversal symmetry, we now derive the equilibrium statistics version of the CISP. In quantum statistics, any physical quantity, say $A$, is expressed in terms of the quantum statistical average $[A]=\operatorname{Tr}(\rho A)$. Adopting the canonical ensemble, the average reads

$$
[A]=\frac{\operatorname{Tr}\left(e^{-\mathcal{H} / k_{B} T} A\right)}{\sum_{\nu} e^{-E_{\nu} / k_{B} T},}
$$

where $k_{B}$ is the Boltzmann constant, $T$ is temperature, $\nu$ is a quantum number labeling the states, and $E_{\nu}$ is the eigenenergy of state $\nu$ solved from Hamiltonian $\mathcal{H}$.

Now consider an unpolarized electron ensemble in a 2DES, subject to Hamiltonian (1). Our main interest here is the CEA of the spin operators of an ensemble of electrons, subject to an identical wave vector $\mathbf{k}$. By this we mean that the summation in Eq. (19) runs over the spin index $\sigma$ only. This gives

$$
[\mathbf{S}]_{\mathbf{k}}=\frac{\hbar}{2} \frac{\operatorname{Tr}\left(e^{-\mathcal{H} / k_{B} T} \overrightarrow{\boldsymbol{\sigma}}\right)}{\sum_{\sigma= \pm} e^{-E_{\sigma}(\mathbf{k}) / k_{B} T}} .
$$

Choosing the basis $|\sigma, \mathbf{k}\rangle$ for the trace, one is led to

$$
[\mathbf{S}]_{\mathbf{k}}=\frac{\hbar}{2} \frac{\sum_{\sigma} e^{-E_{\sigma}(\mathbf{k}) / k_{B} T}\langle\sigma, \mathbf{k}|\vec{\sigma}| \sigma, \mathbf{k}\rangle}{\sum_{\sigma} e^{-E_{\sigma}(\mathbf{k}) / k_{B} T}} .
$$

Using the property (14) and factoring out $e^{-\hbar^{2} k^{2} / 2 m k_{B} T}$ from $e^{-E_{\sigma^{\prime}} / k_{B} T}$, we arrive at the general expression

$$
[\mathbf{S}]_{\mathbf{k}}=-\frac{\hbar}{2} \tanh \frac{\Delta_{\mathbf{k}}}{k_{B} T}\langle+, \mathbf{k}|\vec{\sigma}|+, \mathbf{k}\rangle .
$$

To re-express Eq. (20) in terms of the effective magnetic field $\mathbf{B}_{\text {eff }}(\mathbf{k})$, defined by

$$
\mathbf{B}_{\mathrm{eff}}(\mathbf{k}) \equiv \frac{m c}{e} \Omega(\mathbf{k})=\frac{\frac{\hbar}{2} \Omega(\mathbf{k})}{\mu_{B}},
$$

we rewrite Eq. (12) with $\sigma=+1$ as

$$
\langle+, \mathbf{k}|\vec{\sigma}|+, \mathbf{k}\rangle \cdot \mathbf{B}_{\mathrm{eff}}(\mathbf{k})=\frac{\Delta_{\mathbf{k}}}{\mu_{B}} .
$$

Noting $\langle\sigma, \mathbf{k}|\vec{\sigma}| \sigma, \mathbf{k}\rangle=1$ (unit vector) and $|(\hbar / 2) \Omega(\mathbf{k})|=\Delta_{\mathbf{k}}$, Eq. (22) implies

$$
\langle+, \mathbf{k}|\vec{\sigma}|+, \mathbf{k}\rangle=\hat{\mathbf{B}}_{\mathrm{eff}}(\mathbf{k}),
$$

i.e., the direction of the effective magnetic field. Therefore, Eq. (20) can be written as

$$
[\mathbf{S}]_{\mathbf{k}}=-\frac{\hbar}{2} \tanh \frac{\Delta_{\mathbf{k}}}{k_{B} T} \hat{\mathbf{B}}_{\mathrm{eff}}(\mathbf{k}),
$$

which is exactly the analog of the CEA of electron spin in vacuum subject to an applied magnetic field. ${ }^{26}$

Equation (24) now has a transparent meaning: In the presence of SO coupling, an ensemble of rest electrons $(\mathbf{k} \rightarrow 0)$ is unpolarized since $\Delta_{\mathbf{k} \rightarrow 0}=0$ while it becomes spin-polarized antiparallel to $\hat{\mathbf{B}}_{\text {eff }}(\mathbf{k})$ when moving along $\mathbf{k}$. This picture is schematically shown in Fig. 1. Moreover, the hyperbolic tangent factor $\tanh \left(\Delta_{\mathbf{k}} / k_{B} T\right)$ clearly predicts the decrease with $T$ and the increase with $\Delta_{\mathbf{k}}$ in the polarization magnitude, and therefore explains two signatures of the CISP qualitatively: (i) The CISP may persist up to the room temperature. Taking $\Delta_{\mathbf{k}} \approx 3.68 \mathrm{meV}$ from Ref. 14, one has $\tanh \left[\Delta_{\mathbf{k}} /\left(k_{B}\right.\right.$ $\times 300 \mathrm{~K})] / \tanh \left[\Delta_{\mathbf{k}} /\left(k_{B} \times 10 \mathrm{~K}\right)\right] \approx 14 \%$. (ii) As $\langle\mathbf{k}\rangle \propto V_{0}$ (Ref. 13) implies $\Delta_{\mathbf{k}} \propto V_{0}$, the magnitude of the CISP governed by $\tanh \left(\Delta_{\mathbf{k}} / k_{B} T\right)$ is supposed to increase with the bias, as is experimentally proven. ${ }^{12}$

\section{Explicit forms of current-induced spin polarization}

From Eq. (24), it is now clear that the direction of the CISP is given by the effective magnetic-field direction $\hat{\mathbf{B}}_{\mathrm{eff}}(\mathbf{k})$. Alternatively, one can use the direction of the Larmor frequency vector, $\hat{\Omega}(\mathbf{k})$, to describe the CISP direction since $\mathbf{B}_{\text {eff }}(\mathbf{k})$ and $\vec{\Omega}(\mathbf{k})$ are, by definition of Eq. (21), collinear. Therefore, the CISP direction in III-V bulk semiconductors is given by Eq. (3).

For 2DES grown along [001] with Dresselhaus terms up to the $k^{3}$, Eq. (4) describes the effective magnetic field shown as Fig. 2, which simulates a 100 - -thick InGaAs quantum well with $\left\langle k_{z}^{2}\right\rangle=3.6 \times 10^{-4} \AA^{-2}$ (Ref. 3). The CISP direction is opposite to the effective magnetic field. Note that in Fig. 2, the field distribution near the central region (small $k$ ) is dominated by the linear term (6) (cf. the right inset of Fig. 3).

In the rest of this paper, we focus on the Rashba and linear Dresselhaus [001] terms. For effects with full SO 


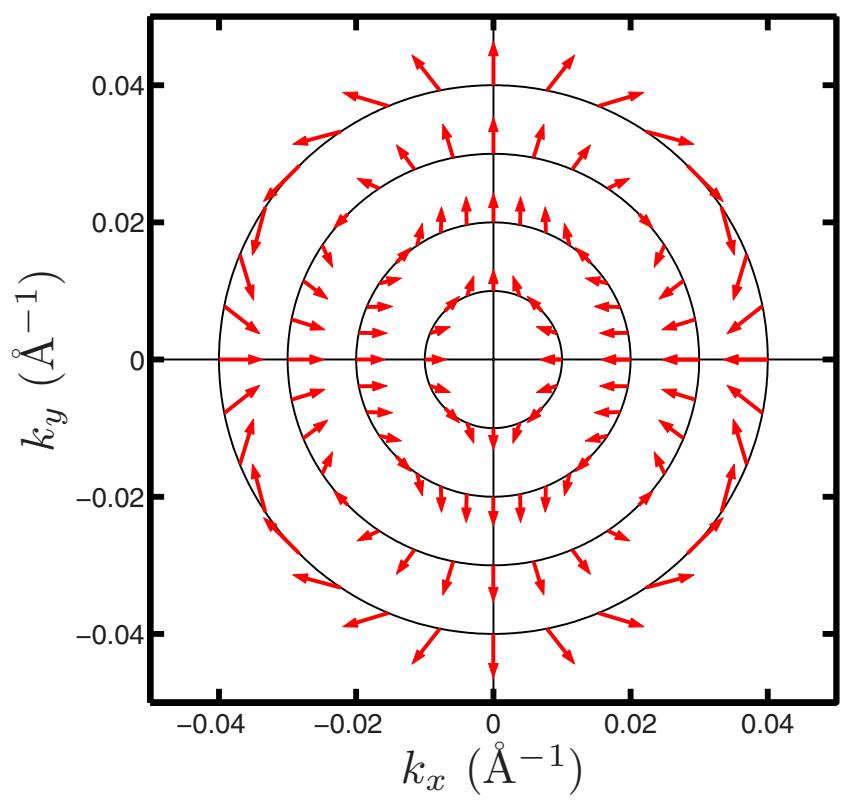

FIG. 2. (Color online) Effective magnetic field of a 100- $\AA$-thick [001] InGaAs quantum well with $\left\langle k_{z}^{2}\right\rangle=3.6 \times 10^{-4} \AA^{-2}$.

terms in the Rashba-Dresselhaus systems, see Refs. 3 and 27. The composite Larmor frequency vector can be obtained by adding Eq. (6) with $\hat{n}=(0,0,1)$ and Eq. (7) together,

$$
\vec{\Omega}_{R D}^{[001]}=\vec{\Omega}_{R}(\hat{n}=\hat{z})+\Omega_{D}^{[001]}=\frac{2}{\hbar}\left[\alpha\left(k_{y},-k_{x}, 0\right)+\beta\left(-k_{x}, k_{y}, 0\right)\right] .
$$

The spin-splitting linear in $k$ takes the form $\Delta_{\mathbf{k}}=|\zeta| k$ with $\zeta$ $=i \alpha e^{-i \phi}+\beta e^{i \phi}$. Thus the CISP in linear Rashba-Dresselhaus [001] 2DESs is explicitly given by

$$
[\mathbf{S}]_{\mathbf{k}}^{R D 001}=-\frac{\hbar}{2} \tanh \frac{|\zeta| k}{k_{B} T} \hat{\Omega}_{R D}^{[001]} .
$$

\section{E. Remark on effective mass}

In general, the in-plane effective mass $m$ of the electrons is not constant but depend strongly on $\mathbf{k}$ for realistic semiconductor systems. However, in the long-wavelength limit $k_{F} a \ll 1\left(k_{F}\right.$ and $a$ the Fermi wave vector and lattice constant, respectively), the effective mass, defined by the inverse of the second derivative of $E(\mathbf{k}) / \hbar^{2}$ with respect to $k$, is a constant due to the parabolic nature of $E(\mathbf{k})$ solved from Hamiltonian (1). In this limit, even though the band structure can be anisotropic due to the interplay between different SO couplings (such as Rashba plus linear Dresselhaus [001]), the effective mass remains constant. In the present analysis, we work in this $k_{F} a \ll 1$ limit, within which the Hamiltonian (1) is valid. Interestingly, our CEA formulas such as Eq. (24) do not contain the dependence of $m$.

Away from $k_{F} a \ll 1$ region, the energy dispersion is no longer parabolic, and the free-electron-like model Hamiltonian (1) and hence the follow-up derivations fail. Analysis of the CISP phenomenon requires other formalisms, such as

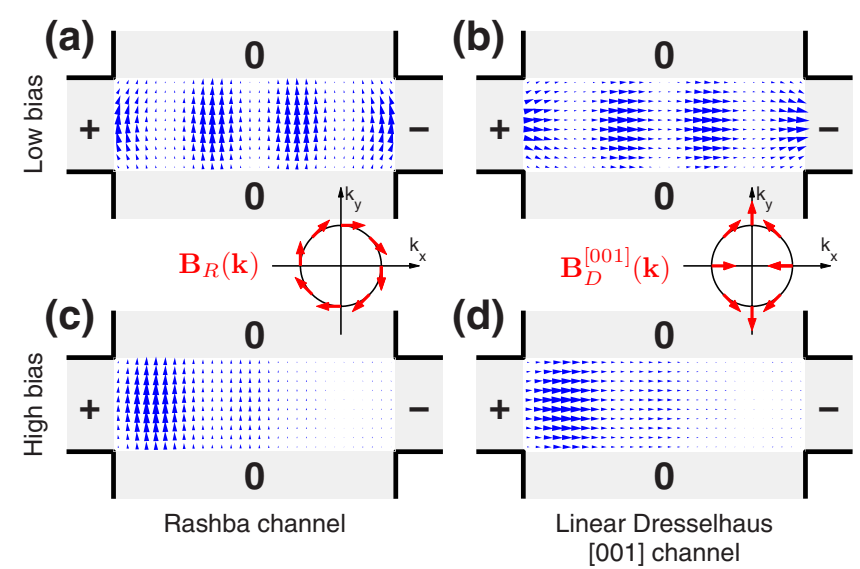

FIG. 3. (Color online) Spin orientation in a $30 a \times 10 a$ channel with $a=1 \mathrm{~nm}$. Channels with linear Rashba model are considered in (a) and (c) while those with linear Dresselhaus [001] model are in (b) and (d). The direction of each sharp triangle represents the inplane spin vector $\langle\mathbf{S}\rangle_{\|}=\left(\left\langle S_{x}\right\rangle,\left\langle S_{y}\right\rangle\right)$ of the local spin density. The size of the triangle depicts the magnitude of $\langle\mathbf{S}\rangle_{\|}$. Effective magnetic fields due to individually the Rashba and the Dresselhaus [001] fields are shown in the insets.

the LKF, to be employed in the coming section. Nevertheless, we will not look further into the influence of the k-dependent effective mass on the CISP.

\section{NUMERICAL RESULTS: LANDAUER-KELDYSH FORMALISM}

To inspect the validity of the previously proposed statistical picture and further examine the CISP, we now perform local spin-density calculation in finite-size 2DESs attached to four normal metal leads by using the LKF. ${ }^{16-18}$

\section{A. Local spin densities in extreme Rashba and Dresselhaus [001] cases}

As a preliminary demonstration, Fig. 3 shows the position-dependent in-plane spin vectors $\langle\mathbf{S}\rangle_{\|}^{\mathbf{r}}=\left(\left\langle S_{x}\right\rangle_{\mathbf{r}},\left\langle S_{y}\right\rangle_{\mathbf{r}}\right)$, with the local spin densities $\left\langle S_{x}\right\rangle_{\mathbf{r}}$ and $\left\langle S_{y}\right\rangle_{\mathbf{r}}$ calculated by the LKF. Here we adopt the finite-difference method and discretize the $30 a \times 10 a$ channel, made of InGaAs/InAlAs heterostructure $^{28}$ grown along [001], into a square lattice with lattice spacing $a=1 \mathrm{~nm}$. Accordingly, this gives the kinetic and Rashba hopping strengths $t_{0} \equiv \hbar^{2} / 2 m a^{2}$ $=0.762 \mathrm{eV}$ and $t_{R} \equiv \alpha / 2 a=3.6 \mathrm{meV}$, respectively. For the Dresselhaus SO coupling, we again assume the quantum well thickness $d=100 \AA$ and $\left\langle k_{z}^{2}\right\rangle \approx(\pi / d)^{2}$, and use $\gamma$ $\approx 27 \mathrm{eV}^{3}$ to give [see Eq. (5)] $\beta=\gamma\left\langle k_{z}^{2}\right\rangle \approx 2.66$ $\times 10^{-2} \mathrm{eV} \AA$, resulting in the Dresselhaus hopping strength $t_{D} \equiv \beta / 2 a=1.33 \mathrm{meV}$.

Let us first consider the extreme cases: pure Rashba and pure Dresselhaus [001] channels. As expected, the spin vectors are mostly oriented antiparallel to $\mathbf{B}_{\mathrm{eff}}(\mathbf{k})$, which is, for $\mathbf{k} \| \hat{x}$, pointing to $-\hat{y}$ in the Rashba channel [Fig. 3(a)/3(c) with low/high bias, respectively] and $-\hat{x}$ in the Dresselhaus [001] channel [Fig. 3(b)/3(d) with low/high bias, respectively]. Here (and hereafter) the low and high biases mean 


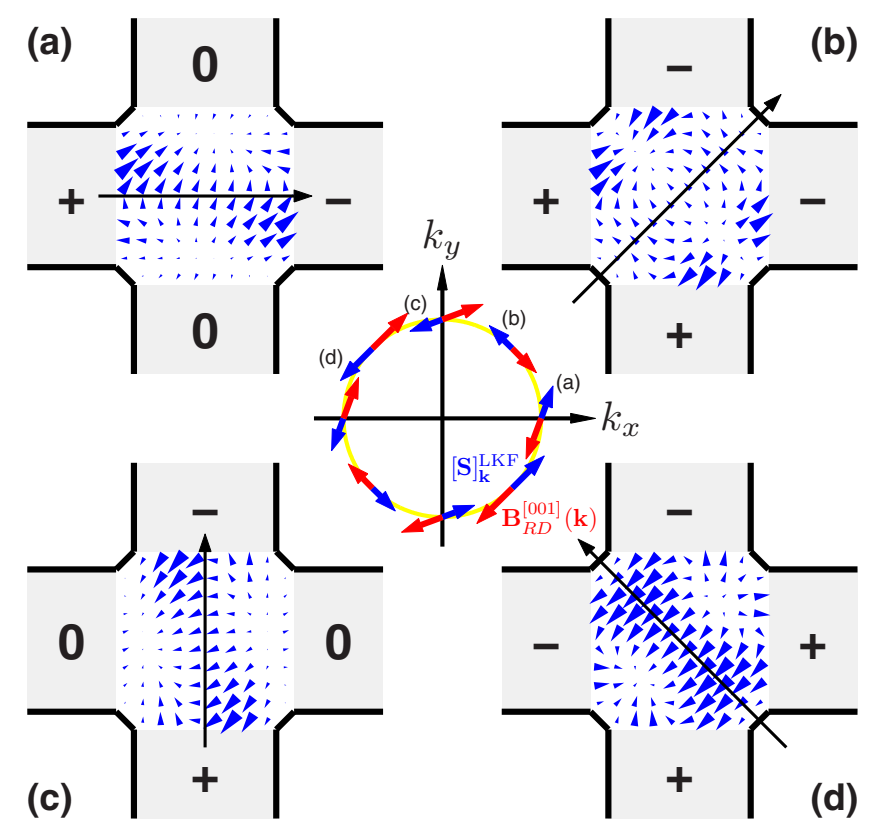

FIG. 4. (Color online) Local spin densities by LKF in a square Rashba-Dresselhaus [001] channel with (a) left to right, (b) left bottom to right top, (c) bottom to top, and (d) right bottom to left top bias configurations. Bias regime belongs to low: $e V_{0}=2 \mathrm{meV}$. Inset: $[\mathbf{S}]_{\mathbf{k}}^{\mathrm{LKF}}$ vs $\mathbf{B}_{R D}^{[001]}(\mathbf{k})$ in the $k_{x}-k_{y}$ coordinate.

$e V_{0}=2 \mathrm{meV}$ and $0.2 \mathrm{eV}$, respectively, and we label the applied potential energy of $\pm e V_{0} / 2$ as " \pm " and $e V_{0}=0$ as " 0 " on each lead. Note that the spin distribution, modulated by the charge distribution, forms standing waves in the low-bias regime since the electrons behave quantum mechanically while that in the high-bias regime, i.e., the nonequilibrium transport regime, decays with distance. ${ }^{19}$ The polarization in the latter (high bias) is about two orders of magnitude stronger than the former (low bias).

\section{B. Consistency check: Analytical canonical ensemble average vs numerical Landauer-Keldysh formalism}

We now consider a four-lead square channel with coexisting Rashba and linear Dresselhaus [001] terms. The coupling constants are set identical to those introduced previously. Removing the four corner sites to avoid short circuit, the sample size is $(10 \times 10-4) a^{2}$. To see if the CISP direction follows the opposite effective magnetic field for all $\mathbf{k}$ directions, we change the current direction by applying different bias configurations. As shown in Figs. 4(a)-4(d), the electrons flow from left to right, from left bottom to right top, from bottom to top, and from right bottom to left top, respectively. Other current directions are done in a similar way but not explicitly shown here. In averaging the in-plane local spin densities $\left\langle S_{x}\right\rangle_{\mathbf{r}}$ and $\left\langle S_{y}\right\rangle_{\mathbf{r}}$ over all the lattice points at $\mathbf{r}$ within the conducting sample, we compare in the inset of Fig. $4[\mathbf{S}]_{\mathbf{k}}^{\mathrm{LKF}} \equiv\left(\overline{\left\langle S_{x}\right\rangle}, \overline{\left\langle S_{y}\right\rangle}\right)$ with the effective magnetic field $\mathbf{B}_{R D}^{[001]}(\mathbf{k})=\left(\hbar / 2 \mu_{B}\right) \vec{\Omega}_{R D}^{[001]}(\mathbf{k})$, where $\vec{\Omega}_{R D}^{[001]}(\mathbf{k})$ is given by Eq. (25). As expected by our statistical picture introduced in Sec. II C, $[\mathbf{S}]_{\mathbf{k}}^{\mathrm{LKF}}$ arrows are all opposite to $\vec{\Omega}_{R D}^{[001]}(\mathbf{k})$ for all $\mathbf{k}$ directions despite some indistinguishably tiny differences.

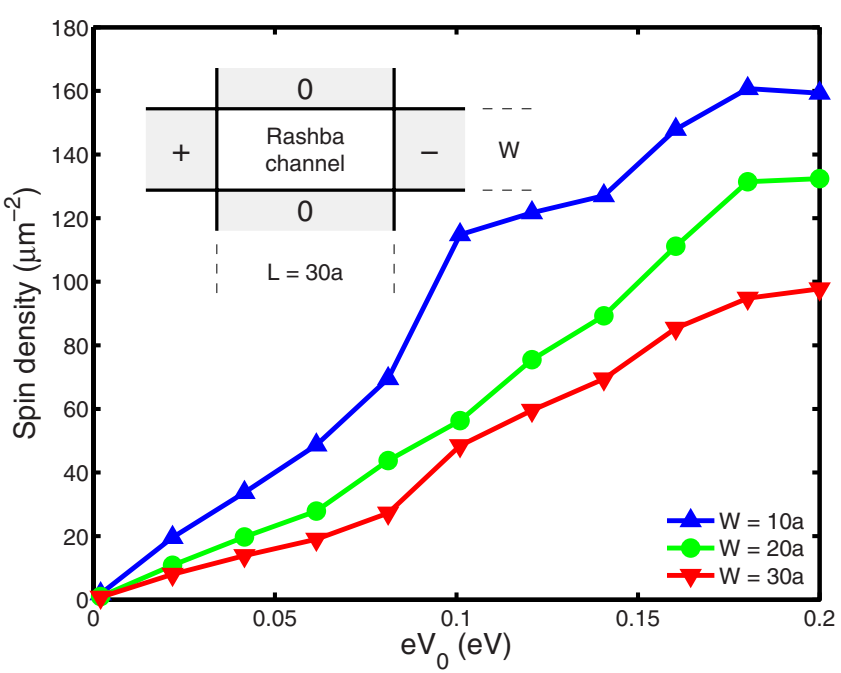

FIG. 5. (Color online) Bias dependence of spin densities induced by the CISP in Rashba 2DESs.

Note that the additive and destructive effects between the two SO terms are also observed at $\pm[\overline{1} 10]$ and $\pm[110]$, respectively. Along $\pm[\overline{1} 10]( \pm[110])$, strongest (weakest) spin splitting $\Delta_{\mathbf{k}}$ and hence the CISP magnitude [Eq. (24)] occur. Note that here we apply low bias. With high bias the results also agree perfectly with the CEA picture (not shown).

\section{Bias dependence of current-induced spin polarization}

Having shown that the statistical argument indeed works well, we next examine the bias dependence of the CISP, which is expected to be a proportional relation, as has been experimentally observed. ${ }^{12}$ We return to Rashba channels. Spin densities, i.e., the total spin divided by the total area of the conducting channel obtained via $\Sigma_{\mathbf{r}}\left\langle S_{y}\right\rangle_{\mathbf{r}} /\left(N a^{2}\right)$ here with $N$ being the number of total lattice sites in the conducting sample, are reported in Fig. 5 for sample widths $W$ $=10 a, 20 a, 30 a$. Sample length is set $L=30 a$. Consistent to the experiment, the calculated spin densities increase with $e V_{0}$. In addition, linear response within $e V_{0} \lesssim 0.1 t_{0}$ $=0.076 \mathrm{eV}$ is clearly observed in all cases. Nonlinearity enters when $e V_{0}$ grows so that nonequilibrium statistics dominates. Note that the calculated local spin-density distribution satisfies the usual SHE symmetry ${ }^{18}$ so that we have $\Sigma_{\mathbf{r}}\left\langle S_{x}\right\rangle_{\mathbf{r}}$ $=\Sigma_{\mathbf{r}}\left\langle S_{z}\right\rangle_{\mathbf{r}}=0$ and $\left|\Sigma_{\mathbf{r}}\langle\mathbf{S}\rangle_{\mathbf{r}}\right|=\Sigma_{\mathbf{r}}\left\langle S_{y}\right\rangle_{\mathbf{r}}$

\section{Comparison of current-induced spin polarization in semiconductor heterostructures and metal/semimetal surface states}

Next we extend the calculation of the spin density due to the CISP to other materials. In addition to semiconductor heterostructures, 2DESs have been shown to exist also on metal surfaces supported by the surface states. ${ }^{29}$ Due to the loss of inversion symmetry, the metallic surfaces may exhibit Rashba spin splitting as well. ${ }^{30,31}$ Here we consider three samples: $54 \times 18 \mathrm{~nm}^{2}$ InGaAs/InAlAs heterostructure, 14.7 $\times 4.9 \mathrm{~nm}^{2} \mathrm{Au}(111)$ surface, and $16.2 \times 5.4 \mathrm{~nm}^{2} \mathrm{Bi}(111)$ surface. We arrange the lead configuration of all the three 
TABLE I. Summary of effective-mass ratio $m / m_{0}$, Rashba constant $\alpha$, Fermi energy $E_{F}$ (relative to the band bottom $E_{b}$ ), and the calculated spin density due to CISP for a set of materials.

\begin{tabular}{lccc}
\hline \hline Material & $\begin{array}{c}\text { InGaAs/InAlAs } \\
\text { (heterostructure) }\end{array}$ & $\begin{array}{c}\mathrm{Au}(111) \\
(\text { surface } \\
\text { state) }\end{array}$ & $\begin{array}{c}\mathrm{Bi}(111) \\
\text { (surface } \\
\text { state) }\end{array}$ \\
\hline$m / m_{0}$ & 0.050 & 0.251 & 0.340 \\
$\alpha(\mathrm{eV} \AA)$ & 0.072 & 0.356 & 0.829 \\
$E_{F}-E_{b}(\mathrm{eV})$ & 0.108 & 0.417 & 0.083 \\
Reference & 28 & 30 & 34 \\
CISP $\left(10^{-3} \mathrm{~nm}^{-2}\right)$ & 0.240 & 2.742 & 8.382 \\
\hline \hline
\end{tabular}

samples as those in Fig. 3 and apply high bias. The sizes we choose here are to maintain roughly the same lattice site number $N \lesssim 1000$ and keep the length-width ratio $\sim 3$. Note that realistic lattice structure are considered for the surface states [hexagonal for $\mathrm{Au}(111)$ and honeycomb for $\mathrm{Bi}(111)$ bilayer], while finite-difference method based on the longwavelength limit for the heterostructure is adopted. For introductory reviews of those surfaces, see Ref. 32 for noblemetal surfaces, including gold, and Ref. 33 for bismuth surfaces.

Band parameters extracted from experiments and the spin densities calculated by the LKF are summarized in Table I. Clearly, the CISP increases with the Rashba parameter $\alpha$. This suggests that the CISP (and actually also the SHE) should be more observable on these surfaces. The recently discovered $\mathrm{Bi} / \mathrm{Ag}(111)$ surface alloy that exhibits a giant spin splitting $^{35}$ is even more promising but we do not perform calculation for this interesting material here.

\section{E. Application of current-induced spin polarization: Generation of in-plane spin-Hall pattern}

Finally, we propose an experimental setup, as an application of the CISP, to generate an antisymmetric edge spin accumulation in the in-plane component, i.e., an in-plane spin-Hall pattern. For simplicity, let us consider a Rashba 2DES with the parameters for the LKF calculation taken the same as those in Fig. 5. Sample size is about $30 \times 30 \mathrm{~nm}^{2}$. We apply high bias of $e V_{0}=0.2 \mathrm{eV}$ and arrange a special bias configuration.

As shown in Fig. 6(a), unpolarized electron currents are injected from the left and right leads, and are guided to the top and bottom ones. Under such design, the spin accumulation in $\left\langle S_{z}\right\rangle_{\mathbf{r}}$ exhibits merely a vague pattern [see Fig. 6(b)]. Contrarily, the pattern of $\left\langle S_{x}\right\rangle_{\mathbf{r}}$ shows not only antisymmetric edge accumulation in the channel but also magnitude much stronger than the out-of-plane component [see Fig. 6(c)]. This pattern is reasonably expected by the CISP due to the opposite charge flows along $\pm \hat{y}$ at the top and bottom edges, and hence resembles an in-plane SHE.

In determining $\left\langle S_{y}\right\rangle_{\mathbf{r}}$, Fig. 6(d) does not show a rotated pattern from $\left\langle S_{x}\right\rangle_{\mathbf{r}}$ due to the nonequilibrium transport. In the nonequilibrium transport regime, a distance apart from the source leads is required to induce the CISP, and therefore no

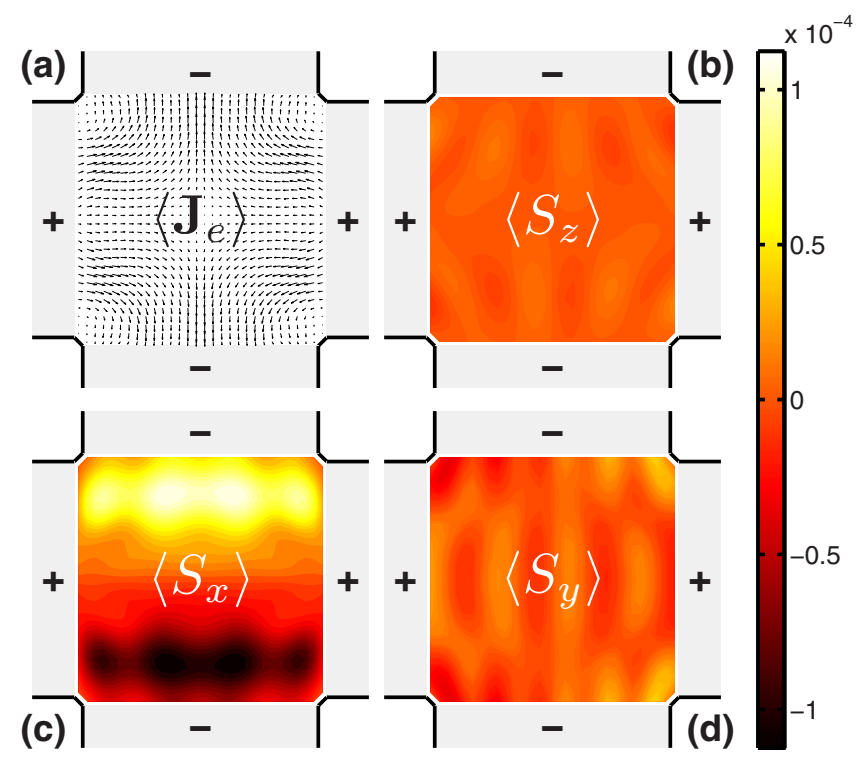

FIG. 6. (Color online) Mapping of the (a) local charge current density, and local spin densities (b) $\left\langle S_{z}\right\rangle$, (c) $\left\langle S_{x}\right\rangle$, and (d) $\left\langle S_{y}\right\rangle$ in a four-terminal square channel with a special bias arrangement. Unit in (b)-(d) is $\hbar / 2$.

significant $\left\langle S_{y}\right\rangle_{\mathbf{r}}$ is observed near the source (left and right) leads. This can be seen by comparing the local spin-density distributions in the low-bias and high-bias regimes shown in Figs. 3(a) and 3(b), and Figs. 3(c) and 3(d), respectively.

\section{SUMMARY}

In conclusion, we have rederived the CISP due to SO coupling in the absence of impurity scattering based on equilibrium statistical mechanics. Correspondingly, a simple picture (Fig. 1) valid for both bulk structures and 2DESs is proposed to help qualitatively explain the CISP. Our explanation for the spin polarization of the moving electron ensemble in solid due to effective magnetic field is an exact analog to that of the rest electron ensemble in vacuum due to external magnetic field. ${ }^{26}$ The picture is further tested to work well even in the regime of nonequilibrium transport in finite-size samples by employing the numerical LKF. Extending the spin-density calculation from the semiconductor heterostructure to metal and semimetal surface states, our calculation confirms that the polarization increases with the SO coupling strength, and hence suggests that the CISP should be more observable on metal and semimetal surfaces with stronger Rashba SO coupling. ${ }^{30,34,35}$ As an application of the CISP, we also suggest an interesting bias configuration for the four-terminal setup to generate in-plane SHE [Fig. $6(\mathrm{c})]$.

\section{ACKNOWLEDGMENTS}

One of the authors (M.H.L.) appreciates S. D. Ganichev and L. E. Golub for stimulating discussions, and L. Ding and G. Bihlmayer for useful information. Financial support of the Republic of China National Science Council Grant No. 952112-M-002-044-MY3 is gratefully acknowledged. 
*Present address: No. 2-1, Fushou Lane, Chengsiang Village, Gangshan Township, Kaohsiung County 82064, Taiwan.

${ }^{1}$ Semiconductor Spintronics and Quantum Computation, edited by D. D. Awschalom, D. Loss, and N. Samarth (Springer, Berlin, 2002).

${ }^{2}$ Y. Kato, R. C. Myers, A. C. Gossard, and D. D. Awschalom, Nature (London) 427, 50 (2004).

${ }^{3} \mathrm{R}$. Winkler, Spin-Orbit Coupling Effects in Two-Dimensional Electron and Hole Systems (Springer, Berlin, 2003).

${ }^{4}$ M. I. D’yakonov and V. Perel', Sov. Phys. JETP 33, 467 (1971).

${ }^{5}$ J. E. Hirsch, Phys. Rev. Lett. 83, 1834 (1999).

${ }^{6}$ S. Murakami, N. Nagaosa, and S. C. Zhang, Science 301, 1348 (2003).

${ }^{7}$ J. Sinova, D. Culcer, Q. Niu, N. A. Sinitsyn, T. Jungwirth, and A. H. MacDonald, Phys. Rev. Lett. 92, 126603 (2004).

${ }^{8}$ Y. K. Kato, R. C. Myers, A. C. Gossard, and D. D. Awschalom, Science 306, 1910 (2004).

${ }^{9}$ J. Wunderlich, B. Kaestner, J. Sinova, and T. Jungwirth, Phys. Rev. Lett. 94, 047204 (2005).

${ }^{10}$ V. M. Edelstein, Solid State Commun. 73, 233 (1990).

${ }^{11}$ A. G. Aronov and Y. B. Lyanda-Geller, JETP Lett. 50, 431 (1989).

${ }^{12}$ Y. K. Kato, R. C. Myers, A. C. Gossard, and D. D. Awschalom, Phys. Rev. Lett. 93, 176601 (2004).

${ }^{13}$ V. Sih, R. C. Myers, Y. K. Kato, W. H. Lau, A. C. Gossard, and D. D. Awschalom, Nat. Phys. 1, 31 (2005).

${ }^{14}$ C. L. Yang, H. T. He, L. Ding, L. J. Cui, Y. P. Zeng, J. N. Wang, and W. K. Ge, Phys. Rev. Lett. 96, 186605 (2006).

${ }^{15}$ N. P. Stern, S. Ghosh, G. Xiang, M. Zhu, N. Samarth, and D. D. Awschalom, Phys. Rev. Lett. 97, 126603 (2006).

${ }^{16}$ S. Datta, Electronic Transport in Mesoscopic Systems (Cambridge University Press, Cambridge, England, 1995).

${ }^{17}$ B. K. Nikolic, S. Souma, L. P. Zarbo, and J. Sinova, Phys. Rev. Lett. 95, 046601 (2005).
${ }^{18}$ B. K. Nikolic, L. P. Zarbo, and S. Souma, Phys. Rev. B 73, 075303 (2006).

${ }^{19}$ M.-H. Liu, G. Bihlmayer, S. Blügel, and C.-R. Chang, Phys. Rev. B 76, 121301(R) (2007).

${ }^{20}$ I. Žutić, J. Fabian, and S. Das Sarma, Rev. Mod. Phys. 76, 323 (2004).

${ }^{21}$ G. Dresselhaus, Phys. Rev. 100, 580 (1955).

${ }^{22}$ M. I. D'yakonov and V. I. Perel, Sov. Phys. JETP 33, 1053 (1971).

${ }^{23}$ W. Knap et al., Phys. Rev. B 53, 3912 (1996).

${ }^{24}$ M. I. D'yakonov and V. Y. Kachorovskii, Sov. Phys. Semicond. 20, 110 (1986).

${ }^{25}$ Y. A. Bychkov and E. I. Rashba, JETP Lett. 39, 78 (1984).

${ }^{26}$ J. J. Sakurai, Modern Quantum Mechanics, revised ed. (Addison-Wesley, New York, 1994).

${ }^{27}$ G. E. Marques, A. C. R. Bittencourt, C. F. Destefani, and S. E. Ulloa, Phys. Rev. B 72, 045313 (2005).

${ }^{28}$ J. Nitta, T. Akazaki, H. Takayanagi, and T. Enoki, Phys. Rev. Lett. 78, 1335 (1997).

${ }^{29}$ S. G. Davison and M. Stęślicka, Basic Theory of Surface States (Oxford University Press, Oxford, UK, 1992).

${ }^{30}$ S. LaShell, B. A. McDougall, and E. Jensen, Phys. Rev. Lett. 77, 3419 (1996).

${ }^{31}$ G. Bihlmayer, Y. M. Koroteev, P. M. Echenique, E. V. Chulkov, and S. Blügel, Surf. Sci. 600, 3888 (2006).

${ }^{32}$ F. Reinert, J. Phys.: Condens. Matter 15, S693 (2003).

${ }^{33}$ P. Hofmann, Prog. Surf. Sci. 81, 191 (2006).

${ }^{34}$ Y. M. Koroteev, G. Bihlmayer, J. E. Gayone, E. V. Chulkov, S. Blügel, P. M. Echenique, and P. Hofmann, Phys. Rev. Lett. 93, 046403 (2004).

${ }^{35}$ C. R. Ast, J. Henk, A. Ernst, L. Moreschini, M. C. Falub, D. Pacile, P. Bruno, K. Kern, and M. Grioni, Phys. Rev. Lett. 98, 186807 (2007). 